\title{
Evaluating the Roomba: A low-cost, ubiquitous platform for robotics research and education
}

\author{
Ben Tribelhorn and Zachary Dodds \\ Department of Computer Science \\ Harvey Mudd College \\ Claremont, California 91711 \\ btribelh@cs.hmc.edu,dodds@cs.hmc.edu
}

\begin{abstract}
This paper presents the iRobot corporation's Roomba vacuum as a low-cost resource for robotics research and education. Sensor and actuation models for unmodified Roombas are presented in the context of both special- and general-purpose spatial-reasoning algorithms, including Monte Carlo Localization and FastSLAM. Further tests probe the feasibility of sensor extensions to the platform. Results demonstrate that, with some caveats, the Roomba is a viable foundation for both classroom and laboratory use, especially for work seeking to leverage robots to other ends, as well as robotics per se with a computational focus.
\end{abstract}

\section{INTRODUCTION}

Perhaps better than any other single platform, iRobot's Roomba vacuum represents ubiquity in robotics today: over two million Roombas are now cleaning floors in homes and other institutions. At US\$150 off-the-shelf, the Roomba is a rugged, autonomous robot that operates in human-scale indoor environments with a sizable proprioceptive sensor suite (Figure 1). As such, it invites applications in domains such as robotics research and education. This paper reports results of both educational and experimental evaluations of the unmodified Roomba platform, including

- the Roomba's commercially available hardware interfaces

- its recently released serial API [1]

- its sensors' and actuators' accuracy

- its support of current spatial-reasoning algorithms

- classroom trials from CS1 and CS2 in spring 2006

- extensibility of its computing and sensory capabilities

The Roomba's vacuuming capabilities comprise a capable set of behaviors. All of them are available as primitives through iRobot's API. Yet these built-in behaviors entirely omit spatial reasoning: no mapping, localization, or other environmental representation is maintained beyond that needed for its reactive decisions. This paper reports our efforts to imbue several unaltered Roombas with these additional, generalpurpose spatial-reasoning capabilities. In the process of using and testing the platform, we have developed software tools for control and visualization, as well as theoretical models of the Roomba's behavior. Thus, this paper's primary technical contributions consist of

- motion and sensor models validated via implementations of Monte Carlo Localization [2] and FastSLAM 1.0 [3]
- comparisons between those models and naive odometry and velocity-integration for spatial reasoning

- platform-specific algorithms for mapping portions of indoor environments

- tested software drivers for Windows, OSX, and Linux

Based on these results and the perspective of eight months of use, we conclude that the Roomba is a promising alternative to the many other low-cost robot platforms available for research and education, particularly for researchers and educators whose focus lies in computational or applied facets of robotics.
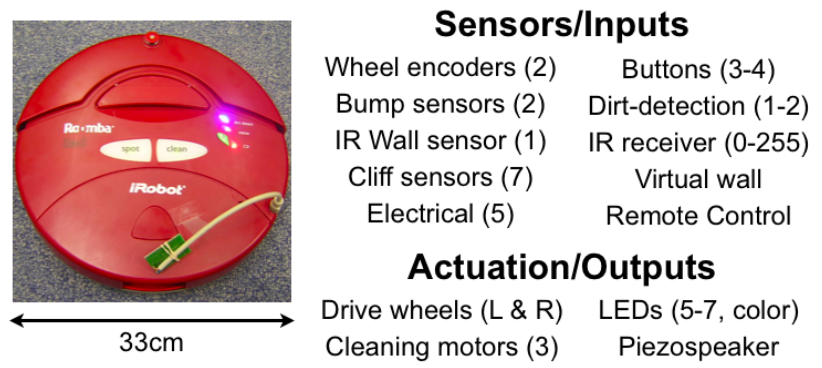

Fig. 1. The Roomba available off-the-shelf for US\$150, along with its built-in sensory and actuation abilities. Proprioception is both capable and complete. Yet it has almost no sensing that reaches beyond the platform itself.

\section{BACKGROUND}

Robotic vacuums are becoming increasingly pervasive. Indeed, vacuuming has been cited as the field's "killer app," bringing more robots into homes than any other [4]. The enabling robotics research spans architectural insights [5], location-aware vacuuming [6], a large number of environmental-coverage algorithms [7], and even outright predictions, now fulfilled [5]. With iRobot's January, 2006 release of the Serial Command Interface (SCI) API to its Roomba platform, there is now an opportunity for robotics researchers and educators to benefit from the successes of the home-robot industry.

This API makes any Roomba an ordinary serial device, though vacuums assembled before 10/2005 require a firmware upgrade. The API specifies a byte-level protocol; this protocol has been incorporated into software drivers written in Java [8], C++ (within Player/Stage) [9], and Python [10]. Even before 
the release of the API, the worldwide community of robot enthusiasts had shown the platform's promise [11] [12]. More recently, Bluetooth, USB, and RS232 serial interfaces have become commercially available [13]. Figure 2 summarizes these devices and their current costs.

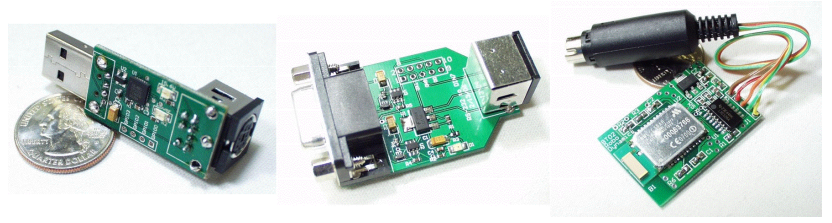

Fig. 2. Commercially available USB, RS232, and Bluetooth serial interfaces to the Roomba provide researchers and educators an inexpensive platform that requires no custom hardware or construction at all. When obtained with a Roomba, these devices are now US\$10, $\$ 5$, and $\$ 80$, respectively [13]

With iRobot-based software support and third-party hardware interfaces, off-the-shelf Roombas are now programmatically accessible without any modification whatsoever. Whimsical applications have emerged: the Roomba has also been adapted to playing the game Frogger on a busy street [14]. But it is not obvious that the Roomba can serve as a resource for validating researchers' algorithms or enabling students to implement and experiment with those algorithms in a lab setting. To our knowledge, this paper's study is the first to address these questions.

\section{COMMUNiCATION AND CONTROL}

\section{A. Hardware}

The most flexible method of communication is Bluetooth which uses the unlicensed $2.4 \mathrm{GHz}$ ISM (Industrial Scientific Medical) band. The aforementioned Bluetooth device (named the RooTooth) is Class 1, allowing a range up to 100 meters. Connection quality over distance drops slowly and our tests indicate that adequate connections can be made up to $60 \mathrm{~m}$. The number of Bluetooth devices that can be used simultaneously is large, as there are 79 channels available. Our tests have demonstrated that a single laptop can easily interact at least five devices concurrently without reducing throughput to individual platforms.

\section{B. Drivers}

We have written two Python layers atop iRobot's byte-level interface. They provide full access to the Roomba's sensors, speaker, motors, and built-in behaviors. As shown in Figure 3, our top layer allows for straight-line translation and includes an odometric correction model.

We have also compared the Bluetooth and USB throughput using this Python layer. USB polling of all of the Roomba's sensors averages an update rate of $66 \mathrm{~Hz}$; Bluetooth is considerably slower. A single Bluetooth module peaks at $16 \mathrm{~Hz}$ in Fast Data Mode and $6 \mathrm{~Hz}$ in its normal mode. A possible solution to this limit in bandwidth would be to mount a microcontroller which reacts to the sensors for stopping quickly and allows higher level decisions to be made by a remote computer which would make higher-level decisions less often.

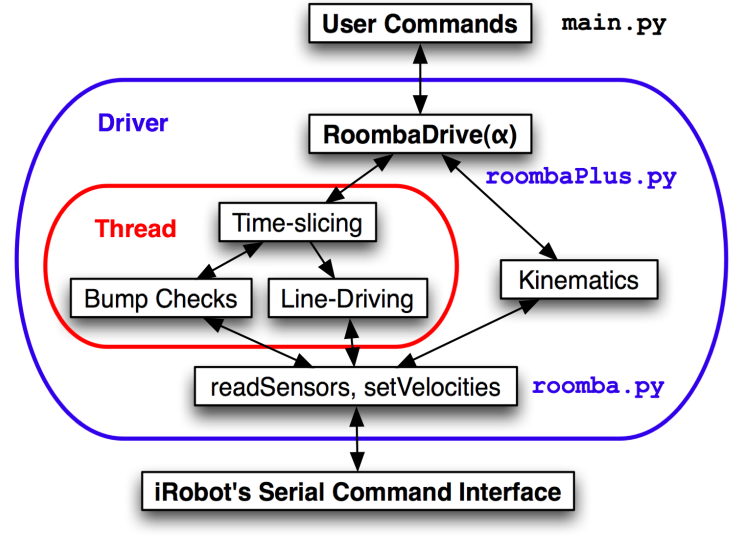

Fig. 3. The basic architecture of our Python driver.

This paper's focus, however, is in maximizing the capabilities of an unaltered Roomba platform.

\section{Simulation}

The Python Robotics (Pyro) toolset already has drivers to access the Roomba's capabilities. In addition, the Roomba is controllable via the player/stage server/simulator.

We have written our own simulator for the Roomba as a test bed for our algorithm implementations. This simple 2D interface enables visualization of all of the Roomba's local sensing and is freely available at [15].

\section{RoOmba Models}

A small number of discrepancies between the Roomba's published programming interface [1] and the platform's behavior motivated our own modeling of its actions and accuracy. For instance, the API cites special codes to indicate straight-line driving (SCI p.4). However, actual behavior did not differ from those commands' large radius of curvature (ROC) limits, published at two meters. Discussions among many experimenters have confirmed the difficulty of straightline motion through the API alone. Second, we found that Roombas within a single production run are quite consistent in their behavior. However, those across different major runs can be very different in their responses to identical API actuation commands under identical environmental conditions.

\section{A. Odometric Modeling}

One of the most important sensing capabilities of the Roomba is odometry. Unfortunately, there is a huge bias when translating left and right and turning around the maximum ROC $\left(80^{\circ}\right.$ vs. $-30^{\circ}$ over $15 \mathrm{~s}$ at $\left.20 \mathrm{~cm} / \mathrm{s}\right)$. A software layer, RoombaDrive, compensates for this bias by allowing simple rotation and linear translation. Linear translation is achieved by time-slicing left and right turns at the maximum ROC. The left-bias time-slicing parameter is denoted $\alpha$, with $0<\alpha<1$. Every half-second, $\alpha$ half-seconds are spent turning left, and $1-\alpha$ half-seconds are spent turning right. 


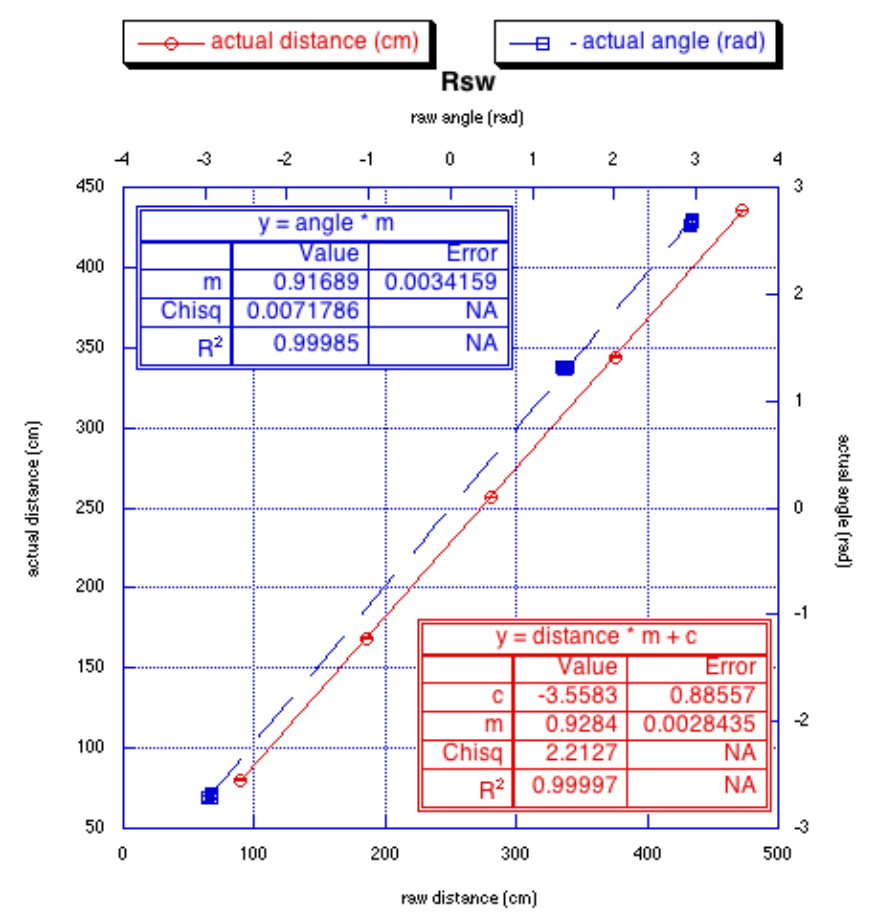

Fig. 4. Regressions of translation and rotation show $m$ to be the same for both of these types of motion for a specific Roomba, named Rsw. We found equations for distance and angle by integrating data from multiple robots, including robots from three different production runs.

Running various tests of our line driving and turning code allowed us to extract a motion model. In Figure 4, data from a single robot is depicted. Linear regressions of raw data versus actual position in linear translation and rotation shows that the slope $m$ is constant between line driving and turning. We ran the tests over multiple robots and production runs and found this correlation to be consistent.

We attempted to find a single robot-specific parameter to allow the calculation of $m$. It turned out that $\alpha$, the lean to the left or right as described above, sufficed for this purpose. First, we ascertained $\alpha$ by time-slicing each robot's largest ROC under various $\alpha$ values until we find one that results in straight-line translation. We then optimize a constrained quadratic relationship (Figure 5) to find a mapping from this $\alpha$ to $m$ summarized in equation 1 . The intercept value of $c=-5$ represents an empirically-tuned correction for the "wobble" inherent in this method of straight-line translation. Like the relationship between $\alpha$ to $m$, this value did not vary among different platforms.

$$
m=0.705+\alpha-\alpha^{2}
$$

Using equation 1, equations for distance and angle $(r, \theta)$ as functions of the raw data provided by the serial API [1] and the robot specific $\alpha$ become the following:

$$
r=\frac{\text { distance }}{10.0} *\left(0.705+\alpha-\alpha^{2}\right)-c
$$

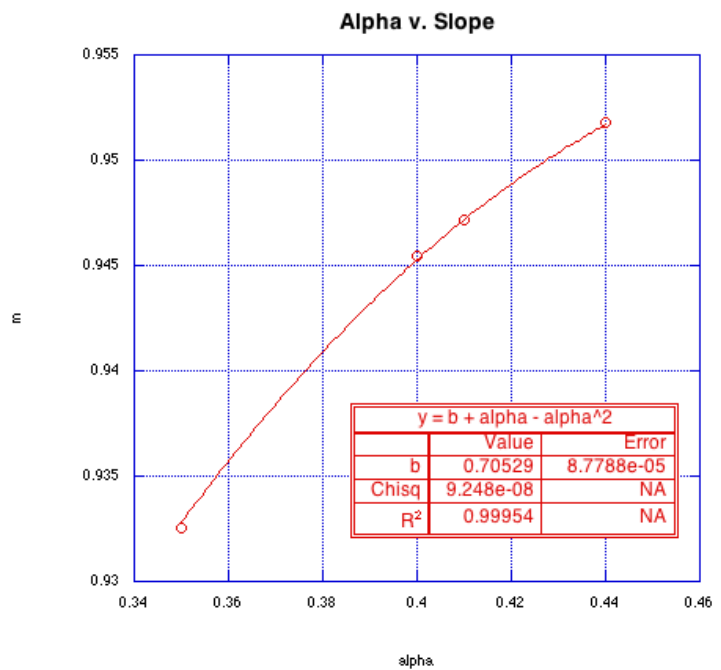

Fig. 5. Iterated regression of empirical data which provides equation 1.

$$
\theta=\frac{\text { angle }}{129.0} *\left(0.705+\alpha-\alpha^{2}\right)
$$

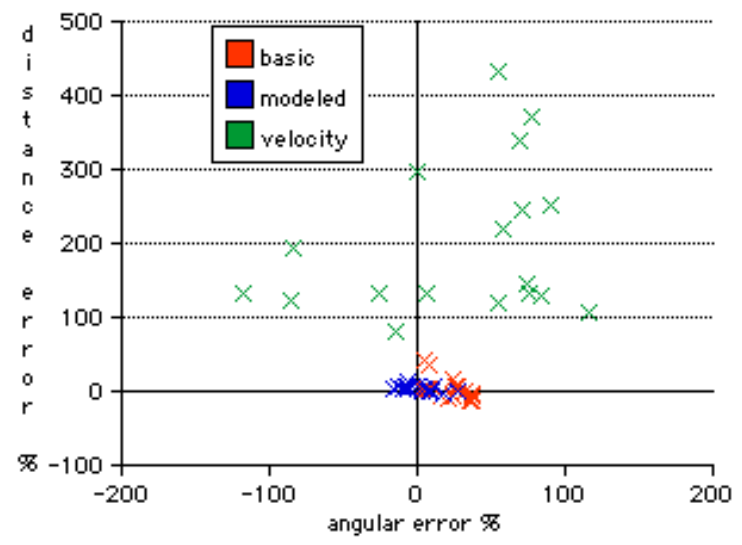

Fig. 6. Comparison of basic kinematic odometry, velocity estimated odometry, and our modeled odometry on two robots from separate batches For this sample $N=18$. Figure 7 presents a closer comparison of basic odometry versus modeled odometry.

Because the odometry is presented only incrementally, rapid odometric sampling will reduce the accuracy of these equations, especially for distance. In situations where it is necessary to sample multiple times per second, we remove the compensatory factor $c$ from equation 2. Our working system samples odometry at every change in velocity.

Results from additional testing of our motion model against basic odometry and velocity-based odometry are illustrated in Figures 6 and 7. The sampled error for naive odometry is $\sigma_{r}=11.5 \%$ and $\sigma_{\theta}=12.4 \%$ which depends strongly on the specific robot (and might be correlated with $\alpha$ ). Our corrected model has error of $\sigma_{r}=2.9 \%$ and $\sigma_{\theta}=6.1 \%$, significantly improving odometric accuracy.

One caveat with our model is that tests with a hardwarealtered Roomba (the main vacuum and brushes had been re- 


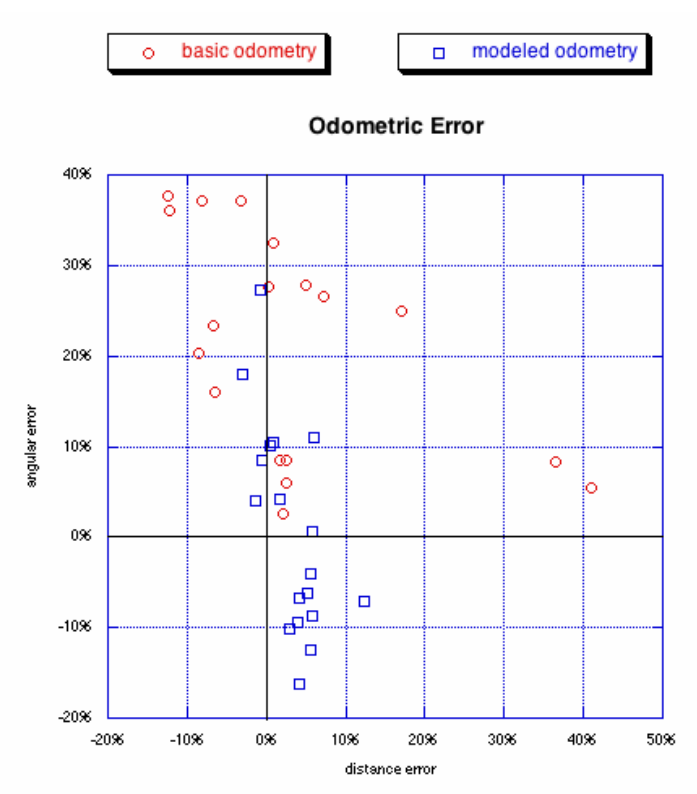

Fig. 7. Here basic odometry is skewed in angular movement $(\bar{x}=21.5 \%)$ and generally far less accurate than our modeled odometry.

moved) showed a larger $m$ value that did not fit equation 1 due to a reduced drag factor. Changing the weight or dynamics of a Roomba thus requires remodeling of the resulting odometry.

\section{B. Sensing Models}

The Roomba only has local sensing in the form of bump and IR sensors. When the Roomba bumps an obstacle, it often slips as it pushes forward, causing the robot to rotate. This rotation is not measured by the odometry. It can be factored into larger error models used by higher-level control or estimation software. The IR sensors can detect virtual walls (provided with the Roomba) and cliffs.

The bump sensors have four states which result from a left and right sensor attached to a single rigid bumper. Thus, these states are left, right, front, or no bump. The collision angle that will produce a front bump varies with the sampling rate. In the ideal, bumps within a forward-facing cone of $\pm 20^{\circ}$ cause both sensors to trigger (due to bumper rigidity). However at sampling rates of around $4 \mathrm{~Hz}$ or less, a front bump will be detected at a range of $\pm 60^{\circ}$ or more. If the response to this bump is similarly slow, the robot will usually slip and actually end its motion facing the wall which can adversely effect odometry. This effect is mitigated, but not eliminated, by our modeled odometry.

\section{Algorithmic VAlidation: MCL And FASTSLAM}

Monte Carlo Localization (MCL) is a probabilistic estimation of pose in a known map that combines range sensing and odometry [2]. Using only the tactile sensing on-board the Roomba we were able to implement and demonstrate successful pose tracking at AAAI 2006. Three snapshots from an example of a successful MCL run are shown in Figure 8; the full video is at [15].
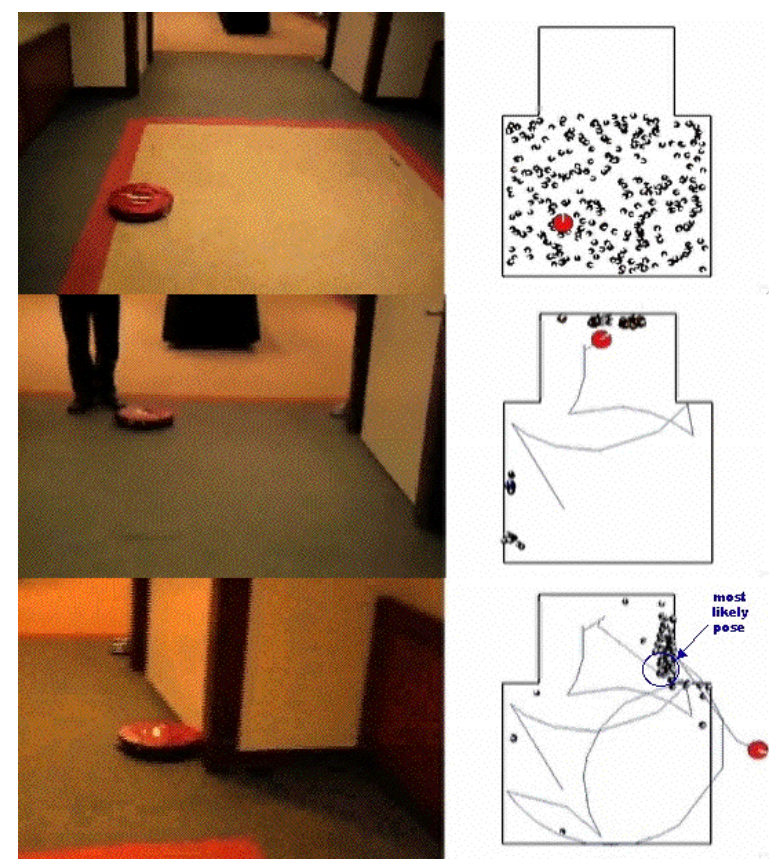

Fig. 8. Recorded at AAAI 2006, this shows a Roomba successfully localizing itself using MCL. In the middle image note that the wall in question is actually an IR-based virtual wall provided with the platform.

Our MCL implementation used an over-generous uniform model of motion error: $35 \%$ in distance and $25 \%$ in angle, in order to compensate for the inaccuracy of the naive kinematic model. Recall that when driving in large arcs the Roomba is extremely biased even while reporting symmetrically identical odometric readings. Thus, this large, uniform error creates a cloud of particles to cover probable locations. Successful runs generally used 300 particles.

Because of many applications' preference for piecewise linear motion, building a motion model of arcs is not always of primary importance. Instead, using the straight-line driving modeled in Section IV, we experimented further with mapping algorithms, as detailed in the next section.

\section{ROOMBA MAPPING}

Mapping with the Roomba presents a stiff challenge. Given the lack of onboard computation and lack of range sensing, mapping is nontrivial. We have designed a preliminary set of mapping algorithms using only local sensing and odometry. What makes them possible is the strong a priori knowledge that we assume:

- A typically indoor, human-scale environment consisting only of walls (and other line segments).

- All such walls and segments are parallel or perpendicular.

These assumptions allow many naive interpretations of the incoming data. For instance, one could fit a line to raw odometry data recorded at each of the Roomba's wall-bumps (the naive algorithm). Our online Roomba-based mapping models each wall as an ellipse and uses our improved model of reported odometry and straight-line driving. Here we present 
our algorithm and contrast the results of our approach in comparison with the naive one.

\section{A. Roomba-specific mapping}

In the general case of $\mathrm{N}$ walls, each bump is a unit of information that is added to our map. We initially begin with one wall. Starting with the second collision we minimize the increase in uncertainty over all walls by limiting the minor axis of the wall to a Roomba's diameter $(33 \mathrm{~cm})$ and the standard deviation of collision angles to 0.5 radians. If the latest bump location does not fit any wall within these threshholds, a new wall is added to the map.

Our results for small runs of eight or fewer bumps align the walls after collecting all the data. Wall alignment uses the angle of the first wall to rotate the second wall and each subsequent wall uses the angle of the previous wall to align itself. This works well for small samples, however around seven or eight collisions corrections to incoming data must be made or else wall uncertainty will become too large and the algorithm will hallucinate erroneous walls.

Thus, to correct larger samples, we begin throwing out old points when fitting new data. This method incorporates enough history to always have a best fit (three points per wall) while stressing the ground truth of each bump reading. The following descriptions contrast the maps made using the naive (raw odometry) and improved (Section IV odometry) models.

Case 1: (single wall) Fitting a line to a set of points is trivial and both the naive and improved odometric models performed well.

Case 2: (a corner) Basic odometry shows slightly better results and less global skew. As is clear in Figure 9, singlecorner finding is not difficult.

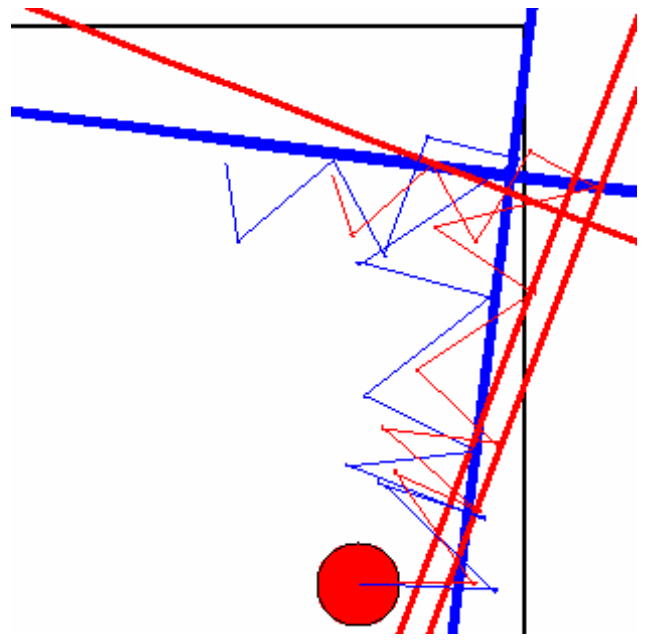

Fig. 9. Blue: Basic odometry. Red: Modeled odometry. Enforcing perpendicularity of walls, both sets of odometric readings find the corner, and both finish close to the true wall-relative location.

Case 3: (a hallway) With a set of five collisions, our odometry model found nearly parallel walls and estimated the distance between them to be $181 \mathrm{~cm}$. The actual distance is $180 \mathrm{~cm}$. Basic odometry, in contrast, estimated that the hallway walls were perpendicular and found three walls; the unconstrained fit is depicted in Figure 11. Figure 12 shows naive odometry from a longer hall run. We are able to find parallel walls as illustrated in Figure 13. With the large data sample correction, our modeled odometry estimates the hallway to be $184.8 \mathrm{~cm}$ and naive odometry estimates $201.4 \mathrm{~cm}$, again with a true $180 \mathrm{~cm}$ distance. Complete results are listed in Table 10. Our modeled odometry - not designed for the purpose of mapping but for the purpose of straight-line translation demonstrates a significant improvement in accuracy.

\begin{tabular}{|c|c|c|}
\hline Line fitting & Modeled & Naive \\
\hline short run & $181.0 \pm 3.0 \mathrm{~cm}$ & $194.1 \pm 101.9 \mathrm{~cm}^{*}$ \\
\hline long run & $184.8 \pm 124.1 \mathrm{~cm}$ & $201.4 \pm 40.9 \mathrm{~cm}$ \\
\hline
\end{tabular}

Fig. 10. Center-to-center distances from hallways cases depicted in Figures 11 and 13. The expected hallway distance is $180 \mathrm{~cm}$. Reported error is the average of the minor axes of the ellipses fit to the walls (three most recent points of each wall). ${ }^{*}$ This distance is from the centers of orthogonal walls.

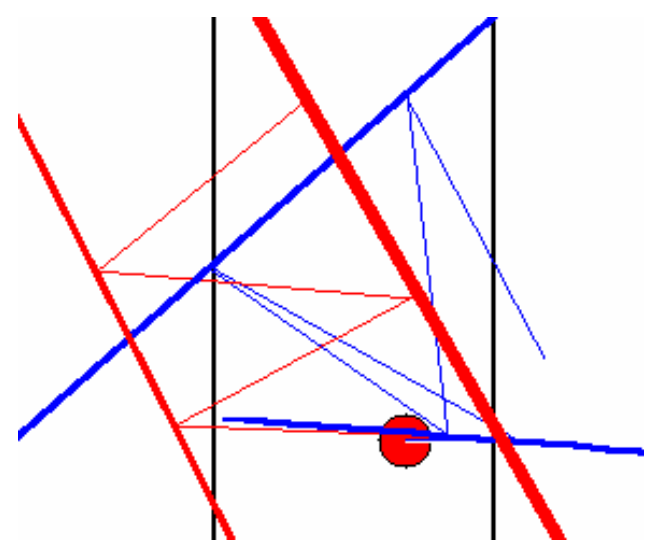

Fig. 11. Unconstrained wall fitting shows modeled odometry to be significantly better than naive odometry.

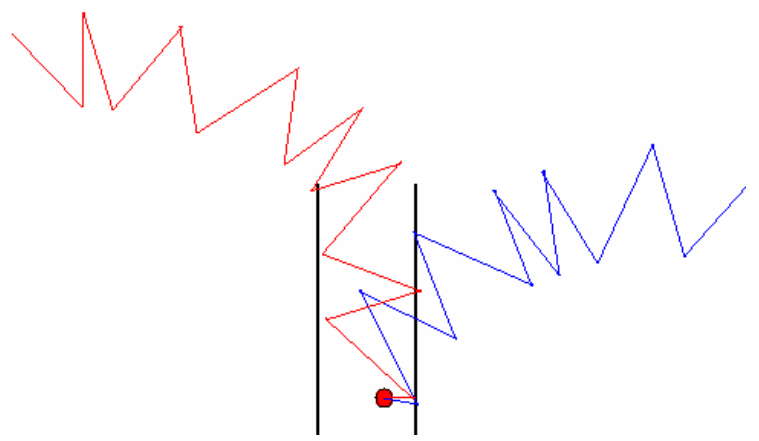

Fig. 12. Here naive odometry is skewed far to the right and our corrected odometry is skewed nearly as much to the left. This run includes 15 bumps in a hallway where the Roomba traveled about 10 meters from its starting location.

\section{B. FastSLAM}

We also implemented FastSLAM 1.0 with known data correspondence as detailed in Probabilistic Robotics. We used a hallway and a simple wandering algorithm to ensure that 


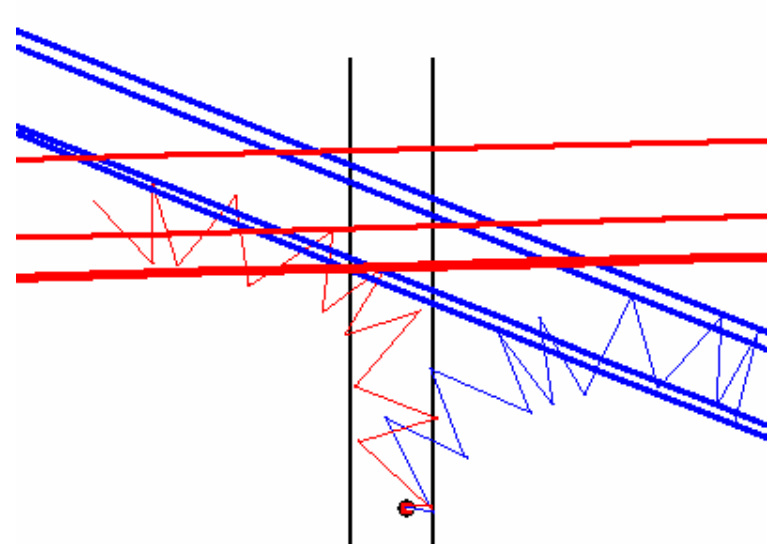

Fig. 13. As relative position is all that matters the correlated hallways have changed position as the odometry continues to gain more error. By only using the last six bumps, a fit can be maintained down a long hallway.

alternating bumps corresponded to each of the two walls, in turn. The walls themselves serve as FastSLAM's landmarks. While a naive adaptation of FastSLAM might ignore the angle of the walls and to assume that the location of the feature is the center of the wall, the results from such a method are not very useful for mapping because they are highly uncertain and do not contain relative angular information.

Thus, our implementation of FastSLAM uses the feature's covariance matrix to model both the angle and uncertainty of a wall, and the point location is then used as the center of the wall. Unfortunately, longer runs suffer from considerable angular drift which degrades the results. As before, only the three most recent points of a wall are used to calculate the uncertainty ellipse. However, unlike our line-fitting algorithm, with FastSLAM the earlier points impact the current ellipses because the movement of each landmark depends on the current covariance at every step. This difference proves to be the key distinction between our algorithm and FastSLAM. To compare the two, we report the single metric parameter estimated by both algorithms, i.e., the estimated distance between the walls. Results from FastSLAM are listed in Table 14.

Not surprisingly, because our alternative approach is so closely coupled with the platform on which it is running, it results in improved accuracy in estimating the inter-wall distance of a hallway. This paper's purpose, however, is not to propose a "correct" mapping algorithm for the Roomba, but to investigate the Roomba's ability to support algorithms of current interest to the community. In addition, the platform scales well pedagogically: it supports probabilistic investigations as ably as it does reactive architectures. Such scalability is unusual in low-cost platforms.

\section{PlatForm EXtensions}

As a result, in conjunction with our odometric models and software drivers, the Roomba is a viable low-cost solution for robotics and AI applications and education. Our algorithmic results demonstrate the ability and promise of this platform.

\begin{tabular}{|c|c|c|}
\hline FastSLAM & Modeled & Basic \\
\hline short run & $158.4 \pm 3.0 \mathrm{~cm}$ & $185.1 \pm 101.9 \mathrm{~cm}$ \\
\hline long run & $99.5 \pm 124.1 \mathrm{~cm}$ & $155.3 \pm 40.9 \mathrm{~cm}$ \\
\hline short (ellipses) & $181.5 \pm 3.0 \mathrm{~cm}$ & $278.6 \pm 101.9 \mathrm{~cm}$ \\
\hline long (ellipses) & $298.7 \pm 124.1 \mathrm{~cm}$ & $570.5 \pm 40.9 \mathrm{~cm}$ \\
\hline
\end{tabular}

Fig. 14. Wall distances from the hallway cases depicted in Figures 11 and 12. The expected hallway distance is $180 \mathrm{~cm}$ and all results save one have this distance within their uncertainty. The best data fit is the short run using corrected odometry and ellipse fitting which is the only one to have a reasonable amount of error. Reported error is the average of the minor axes of the ellipses fit to the walls; given the significant odometric skew for these long runs, large errors are unsurprising.

Yet there are drawbacks: the two most important are the lack of onboard computation (when, as here, it is used as a serial device) and its lack of range sensing. This section describes methods for overcoming these issues.

\section{A. Computation}

A very important feature of any AI device is computational power. Many controllers are available to add this "AI-umph" to the Roomba. PDAs, Gumstix, and micro-controllers are common additions to small mobile robots. One of our solutions is to velcro a laptop to the top of the Roomba. Mounting a laptop on the top the of the robot did not reduce the motors' performance noticeably. At higher speeds, a less precarious solution is to mount a wireless camera on the robot and continue to run the computation off the Roomba itself.

\section{B. Sensing}

Using Mac OS X as this project's primary development environment, we have devloped a $\mathrm{C}$ library that enables access to the YUV pixel values provided by the iSight camera (or any other QuickTime input) [15]. Prior work in this area has not allowed for pixel-level access of values [16]. This development along with the laptop mounted on the Roomba enabled us to implement and run FastSLAM using visual input data with artificial landmarks placed in the environment.

An approach taken by Brian Gerkey [11] for augmenting the Roomba's capabilities adds a laser range finder atop the vacuum's enclosure. This served as the basis for another slam approach, using the algorithms provided as part of Player/Stage. It's noteworthy that the sensor in this case increases the cost of the total system by more than an order of magnitude.

An alternative and very low-cost means to obtain singlepoint range data is to use an onboard camera and a laser pointer, which prior work has shown can provide excellent accuracy for the price (less than $5 \%$ error at $4 \mathrm{~m}$ for US\$40) [17] .

\section{EDUCATIONAL TRIALS}

The Roomba was used as the basis for several assignments in a CS1/CS2 course sequence taught at Chatham College, an all-women's institution in Pittsburgh, PA. Low cost was one reason for choosing the Roomba. Yet a much more important reason was that, as a serial device, the Roomba integrated 
effortlessly into the Python-based environment in which these courses were being taught.

This CS1/CS2 trial included an external assessment effort to determine the extent to which the Roomba (and robots in general) affected students' feelings and capabilities in learning introductory computer science. The results have shown that the physical interactions had a significant impact. One student indicated that the impact was intellectual:

Like when you're just working on the screen it's like 'oh the little dot is moving.' When you're working with the actual thing [the Roomba], you're like okay, problem solving. Because it's a lot easier to solve the problem if it's 3D, in front of you, and you can see exactly what the problem is.

Another student described the robot's impact in affective terms: "Playing with the Roomba made it a lot more fun."

A third student pointed to overcoming some of the Roomba's idiosyncracies when asked Which activities do you think have been most useful this semester in making you a better programmer?:

I would say that probably working with the Roomba definitely just because the first day we worked with it we were trying to get it to go in a straight line because it has like a natural curve to it so it doesn't go straight.

Overall, the Roomba added excitement to the classes, and it provided hands-on, task-specific applications for the programming concepts covered. Moreover, the Roomba did not add the time-intensive overhead of constructing and maintaining Lego-based or other hand-built platforms, nor did it require us to change the programming language or OS on which the class was based. In contrast to many of the other platforms in Figure 15, the Roomba can be used to support an existing CS and $\mathrm{AI}$ curriculum, rather than requiring a curriculum designed especially for it.

\section{Perspective}

These extensions and applications of the Roomba only scratch the surface of what is possible, enabling users an inexpensive basis on which to design systems that run "with our initiation, but without our intervention." [5] As this paper demonstrates, even the ubiquitous, unmodified Roomba platform can support far more than the vacuuming tasks for which it was designed. As an educational resource, the Roomba is pedagogically scalable: it is as suitable for reinforcing beginning programming concepts as it is for exploring algorithms of current interest to the robotics community. As a research resource, the Roomba empowers investigators who want to use robots, rather than build them. For example, it offers researchers involved in the fields of multi-agent systems, HRI, or many other subfields of AI and CS an off-the-shelf means to embody and test their work without having to spend time constructing or modifying hardware.

Ultimately, the Roomba offers the robotics community both an example of the widespread commercial viability of

\begin{tabular}{|c|c|c|}
\hline Platform & Cost & Sensing \\
\hline Lego RCX & $\$ 200$ & Bmp,Lt \\
\hline Roomba & $\$ 230$ & Vis,Mic,Bmp,Enc,WL \\
\hline Lego NXT & $\$ 250$ & Bmp,Lt,Son,Enc,WL \\
\hline Intellibrain & $\$ 300$ & Bmp,Lt,IR,a2d,WL \\
\hline PalmPRK & $\$ 325$ & IR,a2d \\
\hline HandyBoard & $\$ 350$ & Bmp,Lt,IR,a2d \\
\hline KIPR XBC & $\$ 500$ & Vis,Bmp,Lt,IR,Enc \\
\hline UMN eRosi & $\$ 500$ & Lt,Enc,Pyr,WL \\
\hline HandyBoard2 & $\$ 750$ & Vis,Bmp,Lt,IR,Enc,a2d,WL \\
\hline Hemisson & $\$ 780$ & Lt,IR,WL \\
\hline Garcia & $\$ 1725$ & Vis,IR,Enc,WL \\
\hline Khepera & $\$ 2000$ & IR,Enc \\
\hline AIBO & $\$ 2000$ & Vis,Mic,Bmp,Enc,WL \\
\hline
\end{tabular}

Fig. 15. A comparison of several inexpensive robot platforms/controllers, their costs, and their standard set of sensing capabilities. Legend: Bmp, bump or tactile sensing; Lt, light sensing; Vis, vision; Mic, microphone; Enc, encoders or odometry; WL, wireless communication with a controlling PC; a2d, general analog/digital inputs; IR, infrared range sensing; Pyr, heat or flame sensing;

autonomous robots and a novel resource we can leverage toward our educational and research goals. It heralds the advent of robotic peripherals that can take advantage of all of the computing power and cost-efficiency of today's commodity laptop and desktop machines. This paper provides an improved odometric model of the Roomba, some strategies for handling its idiosyncrasies, and and an initial assessment of the Roomba's capabilities. We believe it won't be long before there emerge a wide variety of applications of this modest platform.

\section{ACKNOWLEDGments}

This work was made possible by funds from NSF DUE \#0536173, as well as funding and resources from Harvey Mudd College and Chatham College.

\section{REFERENCES}

[1] Roomba Serial Command Interface (SCI) Specification, iRobot, 2006. [Online]. Available: http://www.irobot.com/hacker

[2] S. Thrun, D. Fox, W. Burgard, and F. Dellaert, "Robust monte carlo localization for mobile robots," Artificial Intelligence, vol. 128, no. 1-2, pp. 99-141, 2001.

[3] M. Montemerlo, S. Thrun, D. Koller, and B. Wegbreit, "FastSLAM: A factored solution to the simultaneous localization and mapping problem," in Proceedings of the AAAI National Conference on Artificial Intelligence. Edmonton, Canada: AAAI, 2002.

[4] L. Grossman, "Maid to order," Time Magazine, vol. September, 2002.

[5] R. Brooks, "Achieving Artificial Intelligence through Building Robots," Massachusetts Institute of Technology, Cambridge, MA, AI-Memo 899, Tech. Rep., 1986.

[6] S. Domnitcheva, "Smart vacuum cleaner - an autonomous location-aware cleaning device," in Adjunct proceedings, Sixth Int. Conf. on Ubiquitous Computing (UbiComp), 2004.

[7] H. Choset, K. Lynch, S. Hutchinson, G. Kantor, W. Burgard, L. Kavraki, and S. Thrun, Principles of Robot Motion: Theory, Algorithms, and Implementations. MIT Press., 2005.

[8] T. Kurt, Hacking Roomba: ExtremeTech. Wiley, 2006, to appear.

[9] B. Gerkey, R. T. Vaughan, and A. Howard, "The player/stage project: Tools for multi-robot and distributed sensor systems," in In Proceedings of the 11th International Conference on Advanced Robotics (ICAR), 2003, pp. 317-323. 
[10] Z. Dodds and B. Tribelhorn, "Erdos: Cost effective peripheral robotics for ai education," in Proceedings, AAAI, 2006, pp. 1966-1967.

[11] B. Gerkey, "Mapping with the iRobot Roomba," 2006. [Online]. Available: http://www.ai.sri.com/ gerkey/roomba/index.html

[12] P. Mecklenburg, "Roomba SLAM," 2005. [Online]. Available: http://www.cs.unc.edu/ prm/roomba/roomba-slam.pdf

[13] "RoombaDevTools," 2006. [Online]. Available: http://www.roombadevtools.com

[14] P. Torrone, "Roomba Tronic," Make Magazine, vol. 06: Robots, 2006.

[15] Z. Dodds and B. Tribelhorn, "Erdos," 2006. [Online]. Available: http://www.cs.hmc.edu/ dodds/erdos

[16] D. Heckenberg, "Using Mac OS X for Real-Time Image Processing," in Proceedings of the Apple University Consortium Conference, 2003.

[17] A. Davidson, B. Tribelhorn, T. Leung, and Z. Dodds, "Low-Cost Range Sensing for Laptop Robots," in Proceedings of the IASTED Conference of Robotics and Applications, 2005, pp. 329-333. 